\title{
Consistent approximation of a nonlinear optimal control problem with uncertain parameters ${ }^{\text {से }}$
}

\author{
Chris Phelps ${ }^{\mathrm{a}}$, Qi Gong ${ }^{\mathrm{a}, 1}$, Johannes O. Royset ${ }^{\mathrm{b}}$, Claire Walton ${ }^{\mathrm{a}}$, Isaac Kaminer ${ }^{\mathrm{c}}$ \\ a Department of Applied Mathematics and Statistics, University of California, Santa Cruz, CA, United States \\ ${ }^{\mathrm{b}}$ Department of Operations Research, Naval Postgraduate School, Monterey, CA, United States \\ ${ }^{\mathrm{c}}$ Department of Mechanical and Astronautical Engineering, Naval Postgraduate School, Monterey, CA, United States
}

\section{A R T I C L E I N F O}

\section{Article history:}

Received 30 April 2013

Received in revised form 6 March 2014

Accepted 11 July 2014

Available online 28 October 2014

Keywords:

Optimal control

Computational methods

Optimization

Nonlinear system

Search theory

\begin{abstract}
A B S T R A C T
This paper focuses on a non-standard constrained nonlinear optimal control problem in which the objective functional involves an integration over a space of stochastic parameters as well as an integration over the time domain. The research is inspired by the problem of optimizing the trajectories of multiple searchers attempting to detect non-evading moving targets. In this paper, we propose a framework based on the approximation of the integral in the parameter space for the considered uncertain optimal control problem. The framework is proved to produce a zeroth-order consistent approximation in the sense that accumulation points of a sequence of optimal solutions to the approximate problem are optimal solutions of the original problem. In addition, we demonstrate the convergence of the corresponding adjoint variables. The accumulation points of a sequence of optimal state-adjoint pairs for the approximate problem satisfy a necessary condition of Pontryagin Minimum Principle type, which facilitates assessment of the optimality of numerical solutions.
\end{abstract}

(c) 2014 Elsevier Ltd. All rights reserved.

\section{Introduction}

In the last decades, a variety of computational algorithms have been developed for solving constrained nonlinear optimal control problems, including Euler (Polak, 1997, chap. 4), Runge-Kutta (Kameswaran \& Biegler, 2008; Schwartz \& Polak, 1996), and Pseudospectral (Gong, Kang, \& Ross, 2006; Kang, 2010; Ross \& Karpenko, 2012). These computational optimal control methods have achieved great success in many areas of control applications (Bedrossian, Bhatt, Kang, \& Ross, 2009; Bedrossian, Karpenko, \& Bhatt, 2012; Chung, Polak, Royset, \& Sastry, 2011; Li, Ruths, Yu, \& Arthanari, 2011). In a standard nonlinear optimal control problem, the objective functional is of the Bolza type, which consists of an end cost as well as an integral over the time domain. In this paper

\footnotetext{
th This work is supported by US Office of Naval Research under Grant N0001412WX21229. The material in this paper was partially presented at the 51st IEEE Conference on Decision and Control (CDC), December 10-13, 2012, Maui, Hawaii, USA. This paper was recommended for publication in revised form by Associate Editor Michael V. Basin under the direction of Editor Ian R. Petersen.

E-mail addresses: cdphelps@soe.ucsc.edu (C. Phelps), qigong@soe.ucsc.edu (Q. Gong), joroyset@nps.edu (J.O. Royset), cwalton@soe.ucsc.edu (C. Walton), kaminer@nps.edu (I. Kaminer).

1 Tel.: +1 831459 3753; fax: +1 8314594482 .
}

we are interested in a class of non-standard optimal control problems in which the objective functional involves an expectation of a Bolza-type cost functional over a space of stochastic parameters. This class of problems is defined in the following.

Problem B. Determine the function pair $\{x, u\}$ with $x \in W_{1, \infty}$ $\left([0,1] ; \mathbb{R}^{n_{x}}\right), u \in L_{\infty}\left([0,1] ; \mathbb{R}^{n_{u}}\right)$ that minimizes the cost functional

$J=\int_{\Omega}\left[F(x(1), \omega)+G\left(\int_{0}^{1} r(x(t), u(t), t, \omega) d t\right)\right] p(\omega) d \omega$

subject to the dynamics

$\dot{x}(t)=f(x(t), u(t))$,

initial condition $x(0)=x_{0}$, and the control constraint $g(u(t)) \leq 0$ for all $t \in[0,1]$.

In Problem $\mathrm{B}, W_{1, \infty}\left([0,1] ; \mathbb{R}^{n_{x}}\right)$ is the space of all essentially bounded functions with essentially bounded distributional derivatives, which map the interval $[0,1]$ into the space $\mathbb{R}^{n_{x}}$, and $L_{\infty}$ $\left([0,1] ; \mathbb{R}^{n_{u}}\right)$ is the set of all essentially bounded functions. The function $p$ is a continuous probability density function for the stochastic parameter $\omega \in \Omega \subset \mathbb{R}^{n_{\omega}}$ and we allow $r$ to be vector valued: that is, $r: \mathbb{R}^{n_{x}} \times \mathbb{R}^{n_{u}} \times \mathbb{R}^{1} \times \mathbb{R}^{n_{\omega}} \mapsto \mathbb{R}^{K}, G: \mathbb{R}^{K} \mapsto \mathbb{R}$. 
Problem B can be viewed as a generalization of the standard nonlinear optimal control problem where the cost function does not involve the stochastic parameter $\omega$. Such a problem formulation allows a broad range of existing control problems to be extended to incorporate parameter uncertainty. For instance, in a number of optimal control applications such as asset protection (Ding, Rahmani, \& Egerstedt, 2009) and target tracking (Quintero, Papi, Klein, \& Chisci, 2010), the objective functional depends on other agents whose behavior may involve parameter uncertainty. Another application which can be addressed using this formulation is optimal path planning in uncertain environments, such as aircraft routing in a threat environment (Zabarankin, Uryasev, \& Pardalos, 2002) or navigating an unmanned surface vehicle in a riverine environment (Gadre, Du, \& Stillwell, 2012). Problem B is also closely related to ensemble control problems studied in, e.g. Ruths and $\operatorname{Li}(2010,2012)$, where the uncertainty appears in both the cost function and the state dynamics.

Our main motivation to study such non-standard optimal control problems is from the topic of optimal search for uncertain targets. Work on search theory can, in general, be divided into two categories depending on how the target is modeled. Mangel (1989) provides a review of the components of the problem and various models used. In the first category, the motion of the target is given by a Markov process. Hellman (1970) and Mangel (1981, 1982) address the problem of computing the posterior distribution of the target's position. Necessary and sufficient conditions for a search plan to be optimal are developed in Hellman (1972), Ohsumi (1991) and Saretsalo (1973). The second category considers targets whose dynamics are conditionally deterministic, which means that the motion of the target depends on a stochastic parameter, and if the value of this parameter is known, the location of the target will be known for each time instance. Such conditionally deterministic targets are considered in Chung et al. (2011), Foraker (2011), Foraker, Royset, and Kaminer (submitted for publication), Lukka (1977), Phelps, Gong, Royset, and Kaminer (2012), Pursiheimo (1976), Royset and Sato (2010) and Sato and Royset (2010), where optimal search plans are given by the solutions to some optimal control problems with objective functionals involving an integral over a space of stochastic parameters, as well as the typical integral over the time-domain. Such optimal search models with conditionally deterministic targets belong to the non-standard optimal control problem considered in this paper, i.e., Problem B.

To briefly demonstrate how the search for conditionally deterministic targets can be modeled as Problem B, consider the problem of a searcher looking for a moving target in order to maximize the probability of detecting the target over some time horizon $[0, T]$ (without loss of generality we assume the time horizon is $[0,1]$ as other time horizons can be handled by rescaling the time parameter). Let the searcher trajectory, $x(t)$, be determined by the dynamical system (1) with initial condition $x_{0}$. We assume that the target's motion is conditionally deterministic. In other words, there exists a random vector $\omega \in \Omega \subset \mathbb{R}^{n_{\omega}}$, such that the trajectory of the target conditioned on $\omega$ is given by $y(\cdot, \omega)$. It is assumed that the probability density of $\omega$ over $\Omega$ is known to the searchers and is given by $p: \Omega \mapsto \mathbb{R}^{+}$. The final component of the search model is a function describing the effectiveness of the searcher. Let $\tilde{r}: \mathbb{R}^{n_{x}} \times \mathbb{R}^{n_{y}} \mapsto \mathbb{R}$ be the instantaneous rate of detection such that the probability of detection in a sufficiently small interval $[t, t+\Delta t]$, conditioned on $\omega$, is given by $\tilde{r}(x(t), y(t, \omega)) \Delta t$. The rate function $\tilde{r}$ is chosen to model the qualities of sensors such as acoustic and sonar sensors. Denote $P(t)$ to be the probability of non-detection at time instance $t$ conditioned on $\omega$. Then

$P(t+\Delta t)=P(t)(1-\tilde{r}(x(t), y(t, \omega)) \Delta t)$.

As $\Delta t \rightarrow 0$ we get

$P(t)=\exp \left(-\int_{0}^{t} \tilde{r}(x(\tau), y(\tau, \omega)) d t\right)$.
Thus the probability that the target is not detected in the time interval $[0,1]$ is given by the integral

$J=\int_{\Omega} \exp \left(-\int_{0}^{1} \tilde{r}(x(t), y(t, \omega)) d t\right) p(\omega) d \omega$.

The problem of finding the trajectory for the searcher which minimizes the probability of not detecting the target can now be framed as a special case of Problem B, with cost functional given by $J[\cdot]$ defined above. Detailed derivation of optimal search models including the construction of detection rate function $\tilde{r}$ can be found in Chung et al. (2011), Foraker (2011) and Foraker et al. (submitted for publication), as well as in Section 5 where an example of an optimal search problem is solved.

Given the difficulty in solving standard nonlinear optimal control problems, it is not surprising that the inclusion of the expectation of the cost functional over the parameter space, combined with the nonlinear dynamics and control constraints, makes Problem B particularly challenging. In the literature, some aspects of Problem $B$ are considered, usually in simplified settings. Early studies into the search problem consider simplified searcher dynamics or conditionally deterministic targets subject to additional special restrictions (Lukka, 1977; Pursiheimo, 1976; Stone, 1977). For example, a necessary condition for optimality is developed in Pursiheimo (1976) for a type of optimal search problem with discrete parameter space. In Lukka (1977), a necessary condition for optimality in the continuous-space setting is derived for a single integrator linear dynamics and a box control constraint. More recent works consider general constrained nonlinear dynamics. In Chung et al. (2011) a numerical algorithm is provided to calculate an optimal solution for a special case of search for a target moving at a constant velocity in a channel. Foraker (2011) and Foraker et al. (submitted for publication) use a composite-Simpson integration scheme to discretize a two-dimensional parameter space and develop a computational method for solving a reduced version of Problem B. Foraker (2011) and Foraker et al. (submitted for publication) also analyze the performance of the computational method using Polak's consistent approximation theory (Polak, 1997, Section 3.3). Ruths and Li (2012) consider an optimal ensemble control problem, which is more general than Problem B in the sense that the uncertain parameter appears in both the cost function and the state dynamics. Consistency and convergence results are developed in Ruths and $\mathrm{Li}$ (2012) for a particular computational method based on a LGL-pseudospectral approximation in both the parameter and time domains.

In this paper we propose a computational framework for the solution of the uncertain optimal control Problem B. Based on the numerical approximation of the integral over the stochastic parameters in the objective functional, the considered uncertain optimal control problem can be approximated by a sequence of standard nonlinear optimal control problems, which can in turn be solved using existing computational methods such as Runge-Kutta (Kameswaran \& Biegler, 2008; Schwartz \& Polak, 1996) and pseudospectral (Gong et al., 2006) approaches. To ensure meaningful results in this computational framework, it is essential to guarantee that the discretization schemes provide valid approximations to the original non-standard optimal control Problem B. Indeed, even for standard optimal control problems, there are counterexamples showing that an inappropriately designed discretization may not be convergent (Cullum, 1972). In this paper, we show that the proposed computational framework approximates the optimal solution to the non-standard optimal control problem under mild assumptions. In particular, we show in Section 3 that the approximation based on the discretization process satisfies a zeroth-order consistency property. That is, accumulation points of a sequence of optimal solutions to the approximate problem are optimal solutions to the original uncertain optimal control problem. We contrast this condition to consistency and convergence results on 
standard optimal control problems, for example results in Gong et al. (2006), Kameswaran and Biegler (2008), Kang (2010) and Polak (1997), as the discretization in this work occurs in the parameter space rather than the time domain. In Section 4 we address the convergence of the corresponding adjoint states, and show that the accumulation points of a sequence of optimal state-adjoint pairs satisfy a necessary condition of Pontryagin Minimum Principle type, which facilities assessment of the optimality of numerical solutions. In Section 5 the proposed computational method is applied to an optimal search problem with constrained nonlinear searcher dynamics and conditionally deterministic target motion. The efficacy of the proposed computational framework is demonstrated through simulations.

\section{Discretization of Problem B}

In this section we present a computational framework for solving the non-standard optimal control Problem B by using a numerical scheme to approximate the integral over the stochastic parameters in the objective functional. The following regularity conditions are assumed.

Assumption 1. The function $g: \mathbb{R}^{n_{u}} \mapsto \mathbb{R}^{n_{g}}$ used in the definition of the control constraint is continuous and the set $U=\left\{v \in \mathbb{R}^{n_{u}} \mid\right.$ $g(v) \leq 0\}$ is compact.

In a real world scenario the set of allowable controls will be bounded and therefore $U$, being a closed and bounded set, will be compact.

Assumption 2. Let $\mathcal{A}$ be the set of feasible pairs to Problem $B$, that is the set of all $\{x, u\}$ with $x \in W_{1, \infty}\left([0,1] ; \mathbb{R}^{n_{x}}\right), u \in L_{\infty}([0,1]$; $\left.\mathbb{R}^{n_{u}}\right)$ such that $u(t) \in U$ and $x(t)=x_{0}+\int_{0}^{t} f(x(s), u(s)) d s$ for all $t \in[0,1]$. Then there exists a compact set $X \subset \mathbb{R}^{n_{x}}$ such that for each feasible pair $\{x, u\} \in \mathcal{A}$ we have $x(t) \in X$ for all $t \in[0,1]$.

This assumption essentially requires for all bounded controls that there is no finite escape time. A large class of nonlinear systems satisfy this assumption, for example, input-to-state stable systems and systems for which $f$ is globally Lipschitz or satisfies a linear growth condition.

Assumption 3. The functions $f, r$ and $G$ are $C^{1}$. The set $\Omega$ is compact. Moreover, for the compact sets $X$ and $U$ defined in Assumptions $1-2$ and for each $t \in[0,1], \omega \in \Omega$, the Jacobian $r_{x}(\cdot, \cdot, t, \omega)$ is Lipschitz on the set $X \times U$, and the corresponding Lipschitz constant is uniformly bounded in $\omega$ and $t$. The function $F(\cdot, \omega)$ is $C^{1}$ on $X$ for all $\omega \in \Omega$; in addition, $F$ and $\nabla_{x} F$ are continuous with respect to $\omega$.

To approximate the integral over the stochastic parameters in the objective functional in Problem B, we introduce numerical integration schemes that satisfy the following assumption.

Assumption 4. For each $M \in \mathbb{N}$, there is a set of nodes $\left\{\omega_{i}^{M}\right\}_{i=1}^{M} \subset$ $\Omega$ and an associated set of weights $\left\{\alpha_{i}^{M}\right\}_{i=1}^{M} \subset \mathbb{R}$, such that for any continuous function $h: \Omega \rightarrow \mathbb{R}$,

$\int_{\Omega} h(\omega) d \omega=\lim _{M \rightarrow \infty} \sum_{i=1}^{M} h\left(\omega_{i}^{M}\right) \alpha_{i}^{M}$.

Throughout the paper, $M$ is used to denote the number of nodes used in the numerical integration scheme. Many numerical integration schemes, e.g., numerical quadrature and Simpson's rule, satisfy Assumption 4 and are applicable to determine the nodes and weights.
Remark 1. Note that if $h_{M}: \Omega \rightarrow \mathbb{R}$ is continuous for all $M \in \mathbb{N}$ and $\left\{h_{M}\right\}$ converges uniformly to $h$, then

$\int_{\Omega} h(\omega) d \omega=\lim _{M \rightarrow \infty} \sum_{i=1}^{M} h_{M}\left(\omega_{i}^{M}\right) \alpha_{i}^{M}$.

This property is frequently used later.

Once the numerical scheme is chosen, the integral over the parameter space is approximated by a sum; and an approximate objective functional for each $M \in \mathbb{N}$ can be defined by

$$
\begin{aligned}
J^{M}= & \sum_{i=1}^{M}\left[F\left(x(1), \omega_{i}^{M}\right)\right. \\
& \left.+G\left(\int_{0}^{1} r\left(x, u, t, \omega_{i}^{M}\right) d t\right)\right] p\left(\omega_{i}^{M}\right) \alpha_{i}^{M} .
\end{aligned}
$$

Now we are ready to define the approximate optimal control problem:

Problem $\mathbf{B}^{\mathbf{M}}$. Determine the function pair $\{x, u\}$, where $x \in W_{1, \infty}$ $\left([0,1] ; \mathbb{R}^{n_{x}}\right)$, and $u \in L_{\infty}\left([0,1] ; \mathbb{R}^{n_{u}}\right)$, that minimizes the cost functional (2) subject to the dynamics (1) and the control constraint $g(u(t)) \leq 0$ for all $t \in[0,1]$.

In Problem $\mathrm{B}^{\mathrm{M}}$, the approximation occurs only in the objective functional; the control and state space of the problem remain the same as in Problem B. The cost functional (2) is not in the standard Bolza form. To facilitate a direct application of Pontryagin Minimum Principle on Problem $B^{\mathrm{M}}$, which will be used in Section 4, it is desirable to transfer the cost functional (2) to standard Bolza form. To this end we introduce the auxiliary variable $z:[0,1] \times \Omega \mapsto \mathbb{R}$ governed by the dynamics

$\dot{z}(t, \omega)=r(x(t), u(t), t, \omega), \quad z(0, \omega)=0, \quad \forall \omega \in \Omega$.

So that $z(1, \omega)=\int_{0}^{1} r(x(t), u(t), t, \omega) d t$. By forming the vector $\zeta_{M}(t)=\left[z\left(t, \omega_{1}^{M}\right), \ldots, z\left(t, \omega_{M}^{M}\right)\right]^{T}$ we can reformulate the objective functional (2) as:

$\hat{J}^{M}=\sum_{i=1}^{M}\left[F\left(x(1), \omega_{i}^{M}\right)+G\left(\zeta_{M, i}(1)\right)\right] p\left(\omega_{i}^{M}\right) \alpha_{i}^{M}$.

This is a Bolza objective functional with an end cost. Therefore Problem $\mathrm{B}^{\mathrm{M}}$ is equivalent to the standard optimal control problem of finding a triplet $\left\{x, \zeta_{M}, u\right\}$ which minimizes the objective functional (4) subject to the dynamics

$\dot{x}(t)=f(x(t), u(t))$

$\dot{\zeta}_{M, i}(t)=r\left(x(t), u(t), t, \omega_{i}^{M}\right), \quad i=1, \ldots, M$

initial condition $x(0)=x_{0}, \zeta_{M, i}(0)=0$, and the control constraint $g(u(t)) \leq 0$ for all $t \in[0,1]$. This formulation is used again when deriving a necessary condition for Problem $B$.

By using a numerical scheme to approximate the integral in the objective functional, the non-standard optimal control Problem $B$ is discretized into a sequence of standard optimal control problems, Problem $\mathrm{B}^{\mathrm{M}}$. Problem $\mathrm{B}^{\mathrm{M}}$ can be solved by existing computational optimal control methods, such as Runge-Kutta (Schwartz \& Polak, 1996), pseudospectral (Gong et al., 2006) methods, and indirect (Betts, 1998; Bryson \& Ho, 1975) type of methods. Also note that, to derive Problem $\mathrm{B}^{\mathrm{M}}$, a variety of numerical integration methods can be used. Therefore, the computational framework developed in this paper constructs a family of approximating optimal control problems, $\mathrm{B}^{\mathrm{M}}$, which can be used to solve the original optimal control Problem B.

\section{Convergence properties of Problem $B^{M}$}

It is well known in computational optimal control that a convergent numerical scheme for solving ODEs may be divergent when 
applied to optimal control problems (Cullum, 1972; Gong et al., 2006; Polak, 1997). Similarly, the convergence of the numerical integration assumed in Assumption 4 does not necessarily imply solutions of Problem $B^{\mathrm{M}}$ converge to solutions of the original Problem $B$. The focus of this section is to show that, under Assumptions $1-4$, accumulation points of a sequence of optimal solutions to the approximate Problem $\mathrm{B}^{\mathrm{M}}$ as the number of nodes $M$ tends to infinity, are optimal solutions to Problem $B$. This consistency property guarantees that Problem $\mathrm{B}^{\mathrm{M}}$ is indeed an appropriate approximation to Problem B.

Before introducing the main convergence result, we first make a note on the notation to be used. We define the set $\mathcal{N}_{\infty}^{\#}=\{V \subset$ $\mathbb{N} \mid V$ infinite . That is, $\mathcal{N}_{\infty}^{\#}$ is the set of all subsequences of $\mathbb{N}$ of infinite length, which are designated by the index set $V \in \mathbb{N}$. When $M \rightarrow \infty$ as usual in $\mathbb{N}$, we write $\lim _{M \rightarrow \infty}$. However, in the case of convergence with respect to a subsequence designated by an index set $V$, we write $\lim _{M \in V}$. For sequences of feasible pairs $\left\{x_{M}, u_{M}\right\}$, the notation $\lim _{M \rightarrow \infty}\left\{x_{M}, u_{M}\right\}=\{x, u\}$ will mean that $\left\{x_{M}, u_{M}\right\}$ converges pointwise to $\{x, u\}$. Similarly $\lim _{M \in V}\left\{x_{M}, u_{M}\right\}=\{x, u\}$ will refer to pointwise convergence of the state-control pair along the subsequence indexed by $V$.

Lemma 1. Suppose that Assumptions 1-3 hold. Then $\mathcal{A}$, the set of feasible pairs to Problem B defined in Assumption 2, is closed in the topology of pointwise convergence.

Proof. Suppose that a sequence $\left\{x_{M}, u_{M}\right\} \subset \mathcal{A}$ and $\lim _{M \rightarrow \infty}\left\{x_{M}\right.$, $\left.u_{M}\right\}=\{x, u\}$. By the continuity of $g, u(t) \in U$ for all $t \in[0,1]$. Note that because $f$ is $C^{1}$, it is Lipschitz continuous on the compact set $X \times U$. Now consider

$$
\begin{aligned}
\| x(t) & -x_{0}-\int_{0}^{t} f(x(s), u(s)) d s \| \\
= & \lim _{M \rightarrow \infty} \| x(t)-\int_{0}^{t} f(x(s), u(s)) d s-x_{M}(t) \\
& +\int_{0}^{t} f\left(x_{M}(s), u_{M}(s)\right) d s \| \\
\leq & \lim _{M \rightarrow \infty} L \int_{0}^{t}\left\|x(s)-x_{M}(s)\right\|+\left\|u(s)-u_{M}(s)\right\| d s \\
& +\left\|x(t)-x_{M}(t)\right\|,
\end{aligned}
$$

where $L$ is the Lipschitz constant of $f$. Because $x(s), x_{M}(s) \in X$ and $u(s), u_{M}(s) \in U$, where $X$ and $U$ are compact, $\left\|x(s)-x_{M}(s)\right\|$ and $\left\|u(s)-u_{M}(s)\right\|$ are bounded for all $s \in[0,1]$ and $M \in \mathbb{N}$. Therefore by the dominated convergence theorem,

$x(t)=x_{0}+\int_{0}^{t} f(x(s), u(s)) d s$

for all $t \in[0,1]$. Hence, $\{x, u\} \in \mathcal{A}$.

Lemma 1 shows that if $\left\{x_{M}, u_{M}\right\}$ is a sequence of feasible pairs for Problem $B$, then any accumulation point of this sequence is a feasible pair. It sets the foundation for the following result.

Theorem 1. Suppose that Assumptions 1-4 hold. In addition, suppose that there exist $V \in \mathcal{N}_{\infty}^{\#}$ and a set of optimal pairs $\left\{x_{M}^{*}, u_{M}^{*}\right\}_{M \in V}$ for Problem $\mathrm{B}^{\mathrm{M}}$ such that

$\lim _{M \in V}\left\{x_{M}^{*}, u_{M}^{*}\right\}=\left\{x^{\infty}, u^{\infty}\right\}$.

Then $\left\{x^{\infty}, u^{\infty}\right\}$ is an optimal solution to Problem B.

Proof. By Lemma $1,\left\{x^{\infty}, u^{\infty}\right\}$ is a feasible solution to Problem B. Next, we prove the optimality of $\left\{x^{\infty}, u^{\infty}\right\}$. From Assumption $3, r$ is bounded and Lipschitz on $X \times U \times[0,1] \times \Omega$ and $G$ is uniformly continuous on $r(X, U,[0,1], \Omega)$. From the Lipschitz continuity of $r$, we have, for all $\omega \in \Omega$

$$
\begin{aligned}
& \int_{0}^{1}\left\|r\left(x_{M}^{*}(t), u_{M}^{*}(t), t, \omega\right)-r\left(x^{\infty}(t), u^{\infty}(t), t, \omega\right)\right\| d t \\
& \quad \leq L \int_{0}^{1}\left\|x_{M}^{*}(t)-x^{\infty}(t)\right\|+\left\|u_{M}^{*}(t)-u^{\infty}(t)\right\| d t .
\end{aligned}
$$

By the dominated convergence theorem,

$\lim _{M \in V} \int_{0}^{1}\left\|x_{M}^{*}(t)-x^{\infty}(t)\right\|+\left\|u_{M}^{*}(t)-u^{\infty}(t)\right\| d t=0$

and this convergence must be uniform in $\omega$. Then by the uniform continuity of $G$ and the continuity of $F$, for each $\epsilon>0$, there must exist $N \in \mathbb{N}$ such that for each $M \in V$ with $M>N$ the following statements hold for all $\omega \in \Omega$

$$
\begin{aligned}
& \| G\left(\int_{0}^{1} r\left(x_{M}^{*}(t), u_{M}^{*}(t), t, \omega\right) d t\right) \\
& \quad-G\left(\int_{0}^{1} r\left(x^{\infty}(t), u^{\infty}(t), t, \omega\right) d t\right) \|<\frac{\epsilon}{2}, \\
& \left\|F\left(x_{M}^{*}(1), \omega\right)-F\left(x^{\infty}(1), \omega\right)\right\|<\frac{\epsilon}{2} .
\end{aligned}
$$

This implies, by the statement in Remark 1,

$\lim _{M \in V} J^{M}\left(x_{M}^{*}, u_{M}^{*}\right)=J\left(x^{\infty}, u^{\infty}\right)$.

Let $\{x, u\}$ be an arbitrary feasible pair for Problem B. Then, based on the optimality of $\left\{x_{M}^{*}, u_{M}^{*}\right\}, J^{M}\left(x_{M}^{*}, u_{M}^{*}\right) \leq J^{M}(x, u)$ for all $M \in V$. Thus

$$
\begin{aligned}
J\left(x^{\infty}, u^{\infty}\right) & =\lim _{M \in V} J^{M}\left(x_{M}^{*}, u_{M}^{*}\right) \\
& \leq \lim _{M \in V} J^{M}(x, u)=J(x, u) .
\end{aligned}
$$

Therefore $\left\{x^{\infty}, u^{\infty}\right\}$ is an optimal pair for Problem B, since it produces the minimum cost among all feasible solutions.

Theorem 1 shows that if a subsequence of optimal solutions to Problem $\mathrm{B}^{\mathrm{M}}$ converges, this limit point is an optimal solution to Problem B. Based on Theorem 1, one can apply existing computational optimal control algorithms to solve Problem $\mathrm{B}^{\mathrm{M}}$. If the solution sequence is observed to be convergent as $M$ increases, then its limit point is an optimal solution to the original non-standard optimal control Problem B.

Remark 2. We refer to an approximation in which accumulation points of a sequence of optimal solutions to the approximate problem are optimal solutions to the original problem as a zeroth order consistent approximation. We contrast this condition to that of Polak (1997, Section 3.3), which in addition requires a condition on stationary points. We note that the consistency property in Theorem 1 differs from the consistency results in Gong et al. (2006), Kameswaran and Biegler (2008), Kang (2010) and Polak (1997) because the discretization occurs in the parameter space instead of the time domain. This results in a sequence of standard optimal control problems which can be further approximated using existing time discretization schemes (Gong et al., 2006; Kameswaran \& Biegler, 2008; Schwartz \& Polak, 1996).

Note that Theorem 1 does not ensure the existence of an accumulation point. However, using a generalized Helly's Selection Theorem from Duchoň and Maličký (2009), we can guarantee the existence of a convergent subsequence for a certain class of controls.

Definition 1 (Duchon̆ $\mathcal{E}$ Maličký, 2009). Let (Y,d) be a metric space and $h:[0,1] \mapsto Y$. A function $h$ is of bounded variation if there 
exists $B>0$ such that for any partition $\pi, 0 \leq t_{0}<t_{1}<\cdots$ $<t_{n}<t_{n+1} \leq 1$, we have $\sum_{i=0}^{n} d\left(h\left(t_{i+1}\right), h\left(t_{i}\right)\right)<B$. The variation of $h$ is defined as

$V_{h}=\sup _{\pi} \sum_{i=0}^{n} d\left(h\left(t_{i+1}\right), h\left(t_{i}\right)\right)$.

We say family $H$ of functions is of uniformly bounded variation if there exists a $C>0$ such that for each $h \in H$, we have $h:[0,1] \mapsto$ $Y$ and $V_{h}<C$.

Corollary 1. Suppose Assumptions 1-4 hold, and in addition there exist $V \in \mathcal{N}_{\infty}^{\#}$ and a set of optimal solutions $\left\{x_{M}^{*}, u_{M}^{*}\right\}_{M \in V}$ to Problem $\mathrm{B}^{\mathrm{M}}$, such that $\left\{u_{M}^{*}\right\}_{M \in V}$ have uniformly bounded variation. Then there exists $V^{\prime} \subseteq V$ such that $\lim _{M \in V^{\prime}}\left\{x_{M}^{*}, u_{M}^{*}\right\}=\left\{x^{\infty}, u^{\infty}\right\}$ for some $\left\{x^{\infty}, u^{\infty}\right\} \in \mathcal{A}$.

Sketch of Proof. Because $\dot{x}=f(x, u)$ and $f$ is bounded on $X \times$ $U,\left\{x_{M}^{*}\right\}_{M \in V}$ is of uniformly bounded variation on $X$. Therefore $\left\{x_{M}^{*}, u_{M}^{*}\right\}$ is of uniformly bounded variation on $X \times U$. Furthermore, $\left\{x_{M}^{*}(t), u_{M}^{*}(t)\right\}_{M \in V}$ is relatively compact, as it is a subset of a compact space. Therefore by the generalization of Helly's Selection Theorem (Duchon̆ \& Maličký, 2009), there exists a $V^{\prime} \subset V$ such that $\lim _{M \in V^{\prime}}\left\{x_{M}^{*}, u_{M}^{*}\right\}=\left\{x^{\infty}, u^{\infty}\right\}$.

It is known that for constrained optimal control problems, the optimal control often belongs to the class of bang-bang controllers, and are piecewise differentiable. If the first derivatives and number of jump discontinuities are bounded, the controls will satisfy the hypothesis in Corollary 1. Therefore the existence of an accumulation point of optimal pairs to Problem $\mathrm{B}^{\mathrm{M}}$ can be guaranteed in this case. From Theorem 1, it is known that this accumulation point is an optimal pair to Problem B.

Remark 3. The reader may notice that we have used pointwise convergence of the state and control to establish the optimality result instead of a weaker condition such as $\mathscr{L}^{p}$ convergence. The result of Theorem 1 can be established using the $\mathcal{L}^{1}$ convergence of the state and control, therefore it will hold under this weaker assumption. However, in this work we focus on the stronger condition of pointwise convergence, as it is necessary to establish the Hamiltonian minimization condition considered in Section 4.

Example 1. We demonstrate the convergence properties on a simplified uncertain optimal control problem for which an analytic optimal solution can be derived. Consider the problem of minimizing the cost functional

$J=\int_{\Omega}\left(\int_{0}^{1} \sum_{k=1}^{K}\left[\left(x_{k}(t)-\omega_{k}\right)^{2}+u_{k}^{2}(t)\right] d t\right) p(\omega) d \omega$,

where $\omega^{T}=\left[\omega_{1}, \ldots, \omega_{K}\right]^{T}$ with $\omega_{k}, k=1,2, \ldots, K$, be independent random variables with joint distribution $p(\omega)$, subject to dynamics $\dot{x}_{k}(t)=u_{k}(t)$, and initial condition $x_{k}(0)=0, k=$ $1,2, \ldots, K$. In optimal search context, this objective function can represent the $K$-dimensional distance to a stationary target at position $\left(\omega_{1}, \omega_{2}, \ldots, \omega_{K}\right)$ with a penalty function $u_{k}^{2}(t)$ intended to keep the control within reasonable bounds.

For parameter $\omega_{k}$, we can assign a set of nodes $\left\{\omega_{k, i}^{M}\right\}_{i=1}^{M}$ and weights $\left\{\alpha_{k, i}^{M}\right\}_{i=1}^{M}$ to approximate the integral over the parameter space based on any numerical integration scheme that satisfies Assumption 4. Remember that the random variables $\omega_{k}$ are independently distributed. We define $p_{k}$ to be the corresponding probability densities, and introduce the following notations

$c_{k}^{M}=\sum_{i=1}^{M} p_{k}\left(\omega_{k, i}^{M}\right) \alpha_{k, i}^{M}, \quad c_{-k}^{M}=\prod_{j \neq k} c_{j}^{M}, \quad c^{M}=\prod_{k} c_{k}^{M}$.
Using these notations, the discretized uncertain optimal control Problem $\mathrm{B}^{\mathrm{M}}$ can be written as: minimizing

$\sum_{k=1}^{K} c_{-k}^{M} \sum_{i=1}^{M}\left[\int_{0}^{1}\left(x_{k}(t)-\omega_{k, i}^{M}\right)^{2}+u_{k}^{2}(t) d t\right] p_{k}\left(\omega_{k, i}^{M}\right) \alpha_{k, i}^{M}$

subject to $\dot{x}_{k}(t)=u_{k}(t), x_{k}(0)=0, k=1,2, \ldots, K$. This is a standard quadratic linear optimal control problem, which can be solved analytically using the Pontryagin Minimum Principle. The closed-form optimal trajectory and control are given by

$x_{k, M}^{*}(t)=\frac{1}{c^{M}} \sum_{i=1}^{M} \omega_{k, i}^{M} p_{k}\left(\omega_{k, i}^{M}\right) \alpha_{k, i}^{M}\left(1-\frac{e^{t}+e^{2-t}}{1+e^{2}}\right)$

$u_{k, M(t)}^{*}=-\frac{1}{c^{M}} \sum_{i=1}^{M} \omega_{k, i}^{M} p_{k}\left(\omega_{k, i}^{M}\right) \alpha_{k, i}^{M} \frac{e^{t}-e^{2-t}}{1+e^{2}}$

From the definition of $c_{k}^{M}, c_{-k}^{M}$ and the convergence of the numerical scheme, we have $\lim _{M \rightarrow \infty} c_{k}^{M}=\lim _{M \rightarrow \infty} c_{-k}^{M}=\lim _{M \rightarrow \infty} c^{M}=$ 1 , and

$\lim _{M \rightarrow \infty} \sum_{i=1}^{M} \omega_{k, i}^{M} p_{k}\left(\omega_{k, i}^{M}\right) \alpha_{k, i}^{M}=\bar{\omega}_{k}$,

where $\bar{\omega}_{k}=\int_{\Omega} \omega_{k} p(\omega) d \omega$. Therefore, the optimal solution of Problem $\mathrm{B}^{\mathrm{M}},\left\{x_{k, M}^{*}, u_{k, M}^{*}\right\}$, has a limit point as $M \rightarrow \infty$, given by

$x_{k}^{*}(t)=\lim _{M \rightarrow \infty} x_{k, M}^{*}(t)=\bar{\omega}_{k}\left(1-\frac{e^{t}+e^{2-t}}{1+e^{2}}\right)$,

$u_{k}^{*}(t)=\lim _{M \rightarrow \infty} u_{k, M}^{*}(t)=-\bar{\omega}_{k} \frac{e^{t}-e^{2-t}}{1+e^{2}}$

According to Theorem 1 , it can be concluded that $x_{k}^{*}$ is the optimal trajectory for the considered non-standard optimal control problem and $u_{k}^{*}$ is the corresponding optimal control. In this example, because the solution to the approximate optimal control Problem $\mathrm{B}^{\mathrm{M}}$ can be given in closed form, it is possible to demonstrate the pointwise convergence of the approximate state and control. In scenarios where the approximate optimal control problem cannot be solved analytically, the pointwise convergence property required in Theorem 1 can be verified numerically.

\section{Convergence in the adjoint variables}

In this section we analyze the convergence of the adjoint variables and Hamiltonian of Problem $\mathrm{B}^{\mathrm{M}}$ and provide a necessary condition which is satisfied by accumulation points of a sequence of optimal solutions. In Section 2 we showed that by introducing an auxiliary vector $\zeta_{M}(t)=\left[z\left(t, \omega_{1}^{M}\right), \ldots, z\left(t, \omega_{M}^{M}\right)\right]^{T}$, where $z$ is given by (3), Problem $B^{\mathrm{M}}$ can be reformulated as a standard optimal control problem with a Bolza cost. It therefore admits the Hamiltonian $\mathbf{H}^{M}: \mathbb{R}^{n_{x}} \times \mathbb{R}^{n_{x}} \times \mathbb{R}^{M} \times \mathbb{R}^{M} \times \mathbb{R}^{n_{u}} \times[0,1] \mapsto \mathbb{R}$ given by

$$
\begin{aligned}
& \mathbf{H}^{M}\left(x, \lambda, \zeta_{M}, \eta_{M}, u, t\right) \\
& \quad=\dot{x}^{T} \lambda+\left[\dot{\zeta}_{M}\right]^{T} \eta_{M} \\
& \quad=[f(x, u)]^{T} \lambda+\sum_{i=1}^{M}\left[r\left(x, u, t, \omega_{i}^{M}\right)\right]^{T} \eta_{M, i},
\end{aligned}
$$

where $\lambda$ and $\eta_{M}$ are the adjoint variables (costates) corresponding to $x$ and $\zeta_{M}$ respectively. By Pontryagin Minimum Principle (Hartl, Sethi, \& Vickson, 1995), if $\left\{x_{M}^{*}, u_{M}^{*}\right\}$ is an optimal solution to Problem $\mathrm{B}^{\mathrm{M}}$, then there exist absolutely continuous costates $\lambda_{M}^{*}$ and $\eta_{M}^{*}$ 
such that the following conditions hold for almost every $t \in[0,1]$ :

$u_{M}^{*}(t) \in \arg \min _{u \in U} \mathbf{H}^{M}\left(x_{M}^{*}(t), \lambda_{M}^{*}(t), \zeta_{M}^{*}(t), \eta_{M}^{*}(t), u, t\right)$,

$\dot{\lambda}_{M}^{*}(t)=-\frac{\partial \mathbf{H}^{M}}{\partial x_{M}^{*}}\left(x_{M}^{*}(t), \lambda_{M}^{*}(t), \zeta_{M}^{*}(t), \eta_{M}^{*}(t), u_{M}^{*}(t), t\right)$,

$\dot{\eta}_{M}^{*}(t)=-\frac{\partial \mathbf{H}^{M}}{\partial \zeta_{M}^{*}}\left(x_{M}^{*}(t), \lambda_{M}^{*}(t), \zeta_{M}^{*}(t), \eta_{M}^{*}(t), u_{M}^{*}(t), t\right)$.

Moreover, the costates satisfy the transversality conditions

$\lambda_{M}^{*}(1)=\frac{\partial \hat{J}^{M}}{\partial x}\left(x_{M}^{*}(1), \zeta_{M}^{*}(1)\right)$,

$\eta_{M}^{*}(1)=\frac{\partial \hat{J}^{M}}{\partial \zeta_{M}}\left(x_{M}^{*}(1), \zeta_{M}^{*}(1)\right)$.

Note that the Hamiltonian (7) and adjoint equation of $\eta_{M}^{*}$ lead to

$\dot{\eta}_{M}^{*}=-\frac{\mathbf{H}^{M}}{\partial \zeta_{M}^{*}}=0$.

Therefore, for all $t \in[0,1]$, we have $\eta_{M, i}^{*}(t)=\eta_{M, i}^{*}(1)$. Thus, from the transversality condition (8) and the objective function (4), we have, for $i=1, \ldots, M$,

$\eta_{M, i}^{*}(t)=\nabla G\left(\zeta_{M, i}^{*}(1)\right) p\left(\omega_{i}^{M}\right) \alpha_{i}^{M}$.

The value, $\zeta_{M, i}(1)$, is given by

$\zeta_{M, i}(1)=z\left(1, \omega_{i}^{M}\right)=\int_{0}^{1} r\left(x(t), u(t), t, \omega_{i}^{M}\right) d t$.

Let $Z$ be the set of all functions from $[0,1] \times \Omega \rightarrow \mathbb{R}^{K}$. We can therefore define an equivalent form of the Hamiltonian, from (7) and (9), so that $\overline{\mathbf{H}}^{M}: \mathbb{R}^{n_{x}} \times \mathbb{R}^{n_{x}} \times \mathbb{R}^{n_{u}} \times Z \times[0,1]$ is given by

$$
\begin{gathered}
\overline{\mathbf{H}}^{M}(x, \lambda, u, z, t)=[f(x, u)]^{T} \lambda+\sum_{i=1}^{M} r\left(x, u, t, \omega_{i}^{M}\right) \\
\cdot \nabla G\left(z\left(1, \omega_{i}^{M}\right)\right) p\left(\omega_{i}^{M}\right) \alpha_{i}^{M} .
\end{gathered}
$$

From this form of the Hamiltonian and the costate dynamics we get the following adjoint equation for $\lambda_{M}^{*}$

$$
\begin{aligned}
\dot{\lambda}_{M}^{*}(t)= & -\left[f_{x}\left(x_{M}^{*}(t), u_{M}^{*}(t)\right)\right]^{T} \lambda_{M}^{*}(t) \\
& -\sum_{i=1}^{M}\left[r_{x}\left(x_{M}^{*}(t), u_{M}^{*}(t), t, \omega_{i}^{M}\right)\right]^{T} \\
& \cdot \nabla G\left(z_{M}^{*}\left(1, \omega_{i}^{M}\right)\right) p\left(\omega_{i}^{M}\right) \alpha_{i}^{M},
\end{aligned}
$$

where $z_{M}^{*}$ is the solution to (3) for the optimal pair $\left\{x_{M}^{*}, u_{M}^{*}\right\}$ and the final value is given by the transversality condition:

$\lambda_{M}^{*}(1)=\sum_{i=1}^{M} \nabla_{x} F\left(x_{M}^{*}(1), \omega_{i}^{M}\right) p\left(\omega_{i}^{M}\right) \alpha_{i}^{M}$.

Now, the necessary condition can be reformulated as:

Necessary Condition of Problem $\mathbf{B}^{\mathbf{M}}$. Suppose that $\left\{x_{M}^{*}, u_{M}^{*}\right\}$ is an optimal pair for Problem $\mathrm{B}^{\mathrm{M}}$. Then $u_{M}^{*}$ must satisfy

$u_{M}^{*}(t) \in \arg \min _{u \in U} \overline{\mathbf{H}}^{M}\left(x_{M}^{*}(t), \lambda_{M}^{*}(t), u, z_{M}^{*}, t\right)$

for almost every $t \in[0,1]$, where $\overline{\mathbf{H}}^{M}$ is given by (10), and $\lambda_{M}^{*}$ is given by (11)-(12) and $z_{M}^{*}$ is the solution to (3) for the pair $\left\{x_{M}^{*}, u_{M}^{*}\right\}$.

We now demonstrate the convergence of the adjoint states $\lambda_{M}^{*}$ and Hamiltonians $\overline{\mathbf{H}}^{M}$. For this purpose, let $\lambda^{\infty}$ be the solution of the initial value problem

$$
\begin{aligned}
\dot{\lambda}^{\infty}(t)= & -\int_{\Omega}\left[r_{x}\left(x^{\infty}(t), u^{\infty}(t), t, \omega\right)\right]^{T} \\
& \cdot \nabla G\left(z^{\infty}(1, \omega)\right) p(\omega) d \omega \\
& -\left[f_{x}\left(x^{\infty}(t), u^{\infty}(t)\right)\right]^{T} \lambda^{\infty}(t), \\
\lambda^{\infty}(1)= & \int_{\Omega} \nabla_{x} F\left(x^{\infty}(1), \omega\right) p(\omega) d \omega,
\end{aligned}
$$

where $z^{\infty}$ is the solution to (3) for the pair $\left\{x^{\infty}, u^{\infty}\right\}$. Furthermore, we define the Hamiltonian of Problem $B$ as $\mathbf{H}: \mathbb{R}^{n_{x}} \times \mathbb{R}^{n_{x}} \times \mathbb{R}^{n_{u}} \times$ $Z \times[0,1]$ such that

$$
\begin{aligned}
\mathbf{H}(x, \lambda, u, z, t)= & {[f(x, u)]^{T} \lambda(t)+\int_{\Omega}[r(x, u, t, \omega)]^{T} } \\
& \cdot \nabla G(z(1, \omega)) p(\omega) d \omega .
\end{aligned}
$$

Remark 4. As opposed to the Hamiltonian used in the optimal control of distributed parameter systems, the Hamiltonian defined in (16) does not explicitly depend on the unknown parameter. This is because the optimal control of Problem $B$ is not a function of the unknown parameter, which is different from the distributed parameter problem.

Theorem 2. Suppose Assumptions 1-4 hold. Let $V \in \mathcal{N}_{\infty}^{\#}$ and let $\left\{x_{M}^{*}, u_{M}^{*}\right\}_{M \in V}$ be a set of optimal solutions to Problem $\mathrm{B}^{\mathrm{M}}$ such that $\lim _{M \in V}\left\{x_{M}^{*}, u_{M}^{*}\right\}=\left\{x^{\infty}, u^{\infty}\right\}$. Let $\lambda_{M}^{*}$ be the solutions to (11)-(12), and $\lambda^{\infty}$ be the solution to (14)-(15). Then for every $t \in[0,1]$

$\lim _{M \in V} \lambda_{M}^{*}(t)=\lambda^{\infty}(t)$.

Moreover, for $\overline{\mathbf{H}}^{M}$ and $\mathbf{H}$ defined in (10) and (16),

$$
\begin{aligned}
& \lim _{M \in V} \overline{\mathbf{H}}^{M}\left(x_{M}^{*}(t), \lambda_{M}^{*}(t), u_{M}^{*}(t), z_{M}^{*}, t\right) \\
& \quad=\mathbf{H}\left(x^{\infty}(t), \lambda^{\infty}(t), u^{\infty}(t), z^{\infty}, t\right) .
\end{aligned}
$$

Proof. Define $\lambda_{M}^{\prime}$ to be the solution of system (11) with final condition (15). Consider the difference

$$
\begin{aligned}
\lambda_{M}^{\prime}(t)-\lambda^{\infty}(t)= & \int_{t}^{1} \dot{\lambda}_{M}^{\prime}(s) d s-\int_{t}^{1} \dot{\lambda}^{\infty}(s) d s \\
= & \int_{t}^{1}\left[f_{x}^{\infty}(s)^{T} \lambda^{\infty}(s)-f_{x}^{M}(s)^{T} \lambda_{M}^{\prime}(s)\right] \\
& +\left[\int_{\Omega} \Gamma^{\infty}(t, \omega) p(\omega) d \omega\right. \\
& \left.-\sum_{i=1}^{M} \Gamma_{M}^{*}\left(t, \omega_{i}^{M}\right) p\left(\omega_{i}^{M}\right) \alpha_{i}^{M}\right] d s
\end{aligned}
$$

where, for notational simplicity, we have defined

$f_{x}^{\infty}(t)=f_{x}\left(x^{\infty}(t), u^{\infty}(s)\right)$,

$f_{x}^{M}(t)=f_{x}\left(x_{M}^{*}(t), u_{M}^{*}(s)\right)$,

$\Gamma^{\infty}(t, \omega)=\left[r_{x}\left(x^{\infty}(t), u^{\infty}(t), t, \omega\right)\right]^{T} \nabla G\left(z^{\infty}(1, \omega)\right)$,

$\Gamma_{M}^{*}(t, \omega)=\left[r_{x}\left(x_{M}^{*}(t), u_{M}^{*}(t), t, \omega\right)\right]^{T} \nabla G\left(z_{M}^{*}(1, \omega)\right)$.

By Assumption $3, f_{X}$ is continuous on the compact set $X \times U$; therefore, $f_{X}\left(x_{M}^{*}(t), u_{M}^{*}(t)\right)$ is uniformly bounded. From (14) it is seen that $\lambda^{\infty}$ is the solution to a system of linear differential equations with bounded coefficients, thus is bounded on the compact domain $[0,1]$. By the dominated convergence theorem

$\lim _{M \in V} \int_{0}^{1} f_{x}^{M}(t)^{T} \lambda^{\infty}(t) d t=\int_{0}^{1} f_{x}^{\infty}(t)^{T} \lambda^{\infty}(t) d t$. 
Similarly, by Assumptions 3-4 and Remark 1, it can be shown that $\lim _{M \in V} \sum_{i=1}^{M} \Gamma_{M}^{*}\left(t, \omega_{i}^{M}\right) p\left(\omega_{i}^{M}\right) \alpha_{i}^{M}=\int_{\Omega} \Gamma^{\infty}(t, \omega) p(\omega) d \omega$.

From these limits, for each $\epsilon>0$, let $M^{\prime} \in \mathbb{N}$ be such that, for every $M \in V$ with $M>M^{\prime}$,

$$
\begin{aligned}
& \int_{0}^{1}\left\|f_{x}^{M}(t)^{T} \lambda^{\infty}(t)-f_{x}^{\infty}(t)^{T} \lambda^{\infty}(t)\right\| d t<\epsilon \\
& \int_{0}^{1} \| \int_{\Omega} \Gamma^{\infty}(t, \omega) p(\omega) d \omega \\
& \quad-\sum_{i=1}^{M} \Gamma_{M}^{*}\left(t, \omega_{i}^{M}\right) p\left(\omega_{i}^{M}\right) \alpha_{i}^{M} \| d t<\epsilon .
\end{aligned}
$$

Therefore, by (17)-(19),

$$
\begin{aligned}
& \left\|\lambda^{\infty}(t)-\lambda_{M}^{\prime}(t)\right\|<\int_{t}^{1}\left\|f_{x}^{M}(s)^{T} \lambda_{M}^{\prime}(s)-f_{x}^{\infty}(s)^{T} \lambda^{\infty}(s)\right\| d s+\epsilon \\
& \leq \int_{t}^{1}\left\|f_{x}^{M}(s)^{T} \lambda_{M}^{\prime}(s)-f_{x}^{M}(s)^{T} \lambda^{\infty}(s)\right\| d s \\
& \quad+\int_{t}^{1}\left\|f_{x}^{M}(s)^{T} \lambda^{\infty}(s)-f_{x}^{\infty}(s)^{T} \lambda^{\infty}(s)\right\| d s+\epsilon \\
& \leq \int_{t}^{1}\left\|f_{x}^{M}(s)^{T} \lambda_{M}^{\prime}(s)-f_{x}^{M}(s)^{T} \lambda^{\infty}(s)\right\| d s+2 \epsilon .
\end{aligned}
$$

Applying Gronwall's inequality gives

$\left\|\lambda^{\infty}(t)-\lambda_{M}^{\prime}(t)\right\| \leq 2 \epsilon \int_{t}^{1} \exp \left\|f_{x}^{M}(s)\right\| d s$.

The function in the integral is uniformly bounded in $M$, and for any $\epsilon>0$ we can find an $S$ such that the statement is valid for each $M \in V, M>S$, thus

$\lim _{M \in V} \lambda_{M}^{\prime}(t)=\lambda^{\infty}(t)$.

Recall that the final conditions, $\lambda_{M}^{*}(1)$ and $\lambda_{M}^{\prime}(1)$ are given by (12) and (15). By Assumption 4 and the continuous dependence of dynamical systems on the initial condition, combined with the convergences $x_{M}^{*}(1) \rightarrow x^{\infty}(1)$ and $\lambda_{M}^{\prime}(t) \rightarrow \lambda^{\infty}(t)$, for each $\epsilon>0$, $t \in[0,1]$ there exists $N \in \mathbb{N}$ such that for each $M>N, M \in V$, the following conditions hold:

$\left\|\lambda_{M}^{*}(t)-\lambda_{M}^{\prime}(t)\right\|<\frac{\epsilon}{2}, \quad\left\|\lambda_{M}^{\prime}(t)-\lambda^{\infty}(t)\right\|<\frac{\epsilon}{2}$.

Therefore

$\lim _{M \in V} \lambda_{M}^{*}(t)=\lambda^{\infty}(t)$.

The proof of the convergence of the Hamiltonians follows a similar argument.

Given the convergence of the adjoint variables and Hamiltonians, we can now show that if the solutions to Problem $\mathrm{B}^{\mathrm{M}}$ have an accumulation point, this accumulation point must minimize the Hamiltonian for Problem B.

Theorem 3. Suppose Assumptions 1-4 hold. Let $V \in \mathcal{N}_{\infty}^{\#}$ and let $\left\{x_{M}^{*}, u_{M}^{*}\right\}$ be a sequence of optimal pairs to Problem $\mathrm{B}^{\mathrm{M}}$ such that $\lim _{M \in V}\left\{x_{M}^{*}, u_{M}^{*}\right\}=\left\{x^{\infty}, u^{\infty}\right\}$. Then there exists an absolutely continuous costate trajectory $\lambda^{\infty}$ satisfying (14)-(15) such that the following holds for almost every $t \in[0,1]$ :

$u^{\infty}(t) \in \arg \min _{u \in U} \mathbf{H}\left(x^{\infty}(t), \lambda^{\infty}(t), u, z^{\infty}, t\right)$

where $\mathbf{H}$ is given by (16) and $z^{\infty}$ is the solution to (3) for the pair $\left\{x^{\infty}, u^{\infty}\right\}$.
Proof. From Theorem $2, \lim _{M \in V} \lambda_{M}^{*}=\lambda^{\infty}$ and

$\lim _{M \in V} \overline{\mathbf{H}}^{M}\left(x_{M}^{*}(t), \lambda_{M}^{*}(t), u, z_{M}^{*}, t\right)=\mathbf{H}\left(x^{\infty}(t), \lambda^{\infty}(t), u, z_{M}^{*}, t\right)$.

Then for any admissable $u \in U$ and each $t \in[0,1]$

$$
\begin{aligned}
& \mathbf{H}\left(x^{\infty}(t), \lambda^{\infty}(t), u^{\infty}(t), z^{\infty}, t\right) \\
& \quad=\lim _{M \in V} \overline{\mathbf{H}}^{M}\left(x_{M}^{*}(t), \lambda_{M}^{*}(t), u_{M}^{*}(t), z_{M}^{*}, t\right) \\
& \leq \lim _{M \in V} \overline{\mathbf{H}}^{M}\left(x_{M}^{*}(t), \lambda_{M}^{*}(t), u, z_{M}^{*}, t\right) \\
& \quad=\mathbf{H}\left(x^{\infty}(t), \lambda^{\infty}(t), u, z^{\infty}, t\right) .
\end{aligned}
$$

In the previous section, Theorem 1 shows that an accumulation point of the set of optimal pairs to Problem $B^{\mathrm{M}}$ is an optimal solution of Problem B. Theorem 3 further provides necessary conditions that such an accumulation point must satisfy. Such results can be applied to verify the optimality of the computed solution. It can also be used to develop algorithms for Problem $B$ by solving the necessary conditions as demonstrated in the following example.

Revisit of Example 1. In the previous section, the analytic optimal solution of Example 1 was obtained by an application of Theorem 1 . Now we show that Theorem 3 provides an alternative way to solve this example problem. First note that in this example, $G(z)=z$, so that $\nabla G(z(1, \omega))=1$. Then from (16) the Hamiltonian of this problem is given by:

$$
\begin{aligned}
& \mathbf{H}(x(t), \lambda(t), z, u(t), t) \\
& \quad=\lambda^{T}(t) u(t)+\sum_{k=1}^{K}\left(x_{k}^{2}(t)+u_{k}^{2}(t)-2 x_{k}(t) \bar{\omega}_{k}+\overline{\omega_{k}^{2}}\right),
\end{aligned}
$$

where $\bar{\omega}_{k}=\int_{\Omega} \omega_{k} d \omega, \overline{\omega_{k}^{2}}=\int_{\Omega} \omega_{k}^{2} d \omega$. Here we use the independence of the random variables $\omega_{k}$ to evaluate the integral over $\Omega$. The costate, $\lambda=\left[\lambda_{1}, \ldots, \lambda_{K}\right]^{T}$, satisfies adjoint equation

$$
\begin{aligned}
& \dot{\lambda}_{k}(t)=-2 x_{k}(t)+2 \bar{\omega}_{k}, \\
& \lambda_{k}(1)=0, \quad k=1, \ldots, K .
\end{aligned}
$$

Because the system is unconstrained, the Hamiltonian minimization condition in Theorem 3 requires

$\frac{\partial \mathbf{H}}{\partial u_{k}}=\lambda_{k}(t)+2 u_{k}(t)=0$,

for $k=1, \ldots, K$. Eqs. (21), (22), together with dynamics, results in a boundary value problem

$$
\begin{gathered}
{\left[\begin{array}{c}
\dot{x}_{k}(t) \\
\dot{\lambda}_{k}(t)
\end{array}\right]=\left[\begin{array}{c}
0 \\
2 \bar{\omega}_{k}
\end{array}\right]+\left[\begin{array}{cc}
0 & -\frac{1}{2} \\
-2 & 0
\end{array}\right]\left[\begin{array}{l}
x_{k}(t) \\
\lambda_{k}(t)
\end{array}\right],} \\
x_{k}(0)=0, \lambda_{k}(1)=0,
\end{gathered}
$$

that can be solved to yield the same optimal solution as shown in (5)-(6). Therefore in this scenario, the necessary condition of Theorem 3 can be used to determine the closed form solution to Problem B.

Similar to Corollary 1 , the following result can be established to ensure the existence of a solution satisfying the condition of Theorem 3.

Corollary 2. Suppose Assumptions 1-4 hold, and in addition there exist $V \in \mathcal{N}_{\infty}^{\#}$ and a set of optimal solutions $\left\{x_{M}^{*}, u_{M}^{*}\right\}_{M \in V}$ to Problem $\mathrm{B}^{\mathrm{M}}$, such that $\left\{u_{M}^{*}\right\}_{M \in V}$ have uniformly bounded variation. Then there exist an optimal solution, $\left\{x^{\infty}, u^{\infty}\right\}$, to Problem B and a costate, $\lambda^{\infty}$, satisfying condition (14), (15) and (20).

Proof. The corollary follows direction from Corollary 1 and Theorem 3. 
a
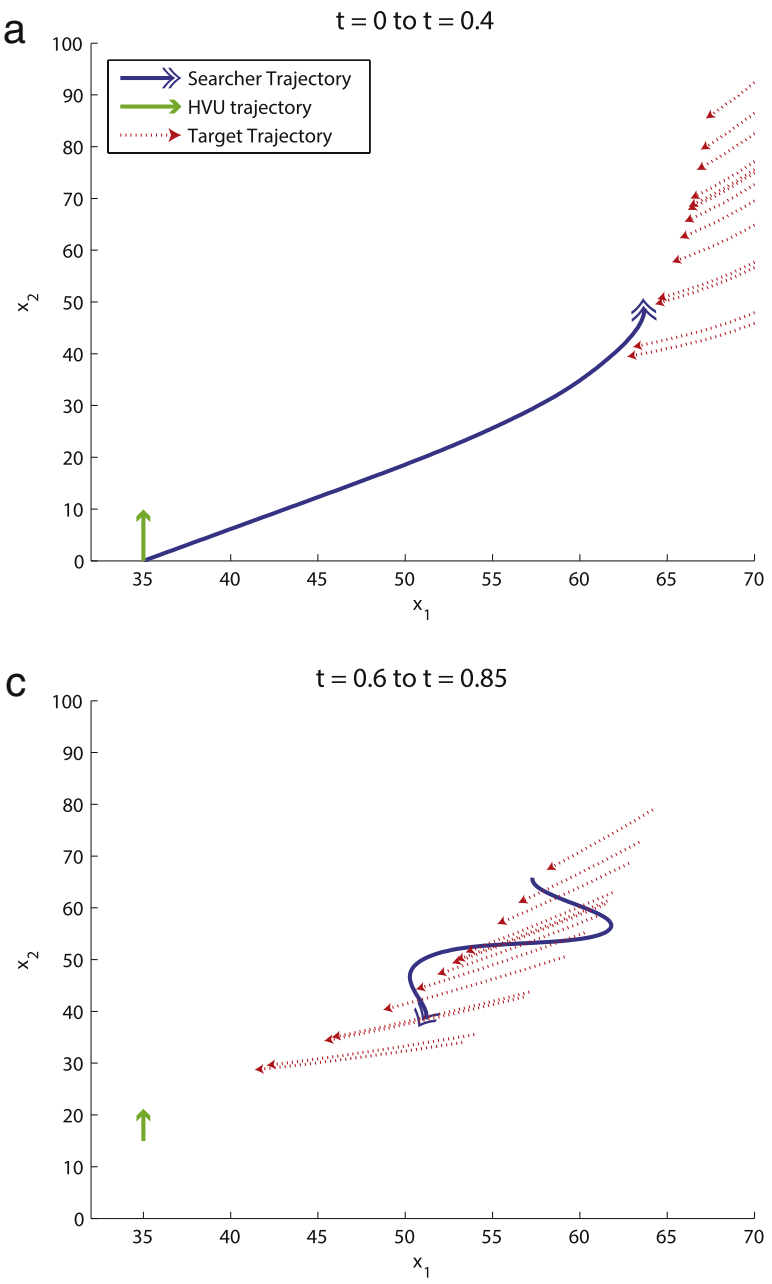
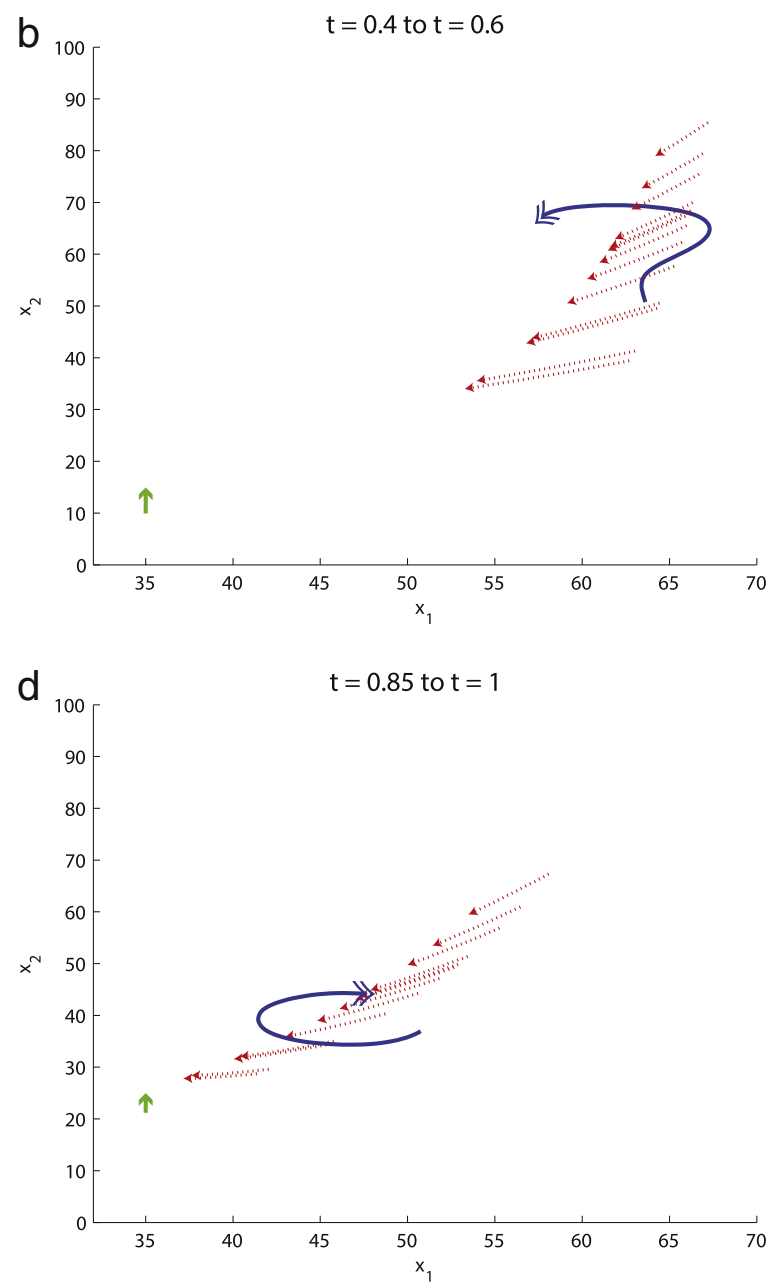

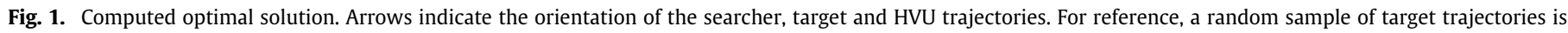
shown, where the initial starting location is determined by a $\operatorname{Beta}(4,2)$ distribution.

\section{Application on optimal search}

In this section, we apply the results of the previous sections to an optimal search problem inspired by a real-world scenario. The example, taken from Foraker (2011) and Foraker et al. (submitted for publication), considers a surface vessel attempting to detect a hostile target with sonar. The target travels towards a friendly ship, called the "high value unit" or "HVU". The objective of the problem is to find a search path that maximizes the chance of detecting the target, before the target reaches the "HVU".

The searcher is modeled as a Dubin's vehicle with dynamics

$\dot{x}_{1}(t)=v \cos x_{3}(t)$,

$\dot{x}_{2}(t)=v \sin x_{3}(t)$,

$\dot{x}_{3}(t)=u(t)$,

where $\left(x_{1}, x_{2}\right)$ represents the position of the searcher and $x_{3}$ is the heading angle. The forward velocity is set to be a constant $v=150$. The control, $u$, is the turning rate of the vehicle that satisfies $|u(t)| \leq 50$ for all $t \in[0,1]$. In the scenario we consider, the HVU travels in the positive $x_{2}$ direction at a constant speed of 25 , and the starting location of the HVU is $(35,0)$. The initial state of the searcher is given by $\left(x_{1}(0), x_{2}(0), x_{3}(0)\right)=\left(35,0, \frac{\pi}{6}\right)$. We assume the trajectory of the target is conditionally deterministic, with starting $x_{1}$ coordinate fixed at 70 and $x_{2}$ coordinate distributed in the domain $[0,100]$ according to a Beta distribution. That is, the starting location of the target is given by $y(0, \omega)=$ $(70, \omega)$ for $\omega \in[0,100]$ and $p(\omega)=p_{4,2}(\omega / 100)$, where $p_{\alpha, \beta}$ is the

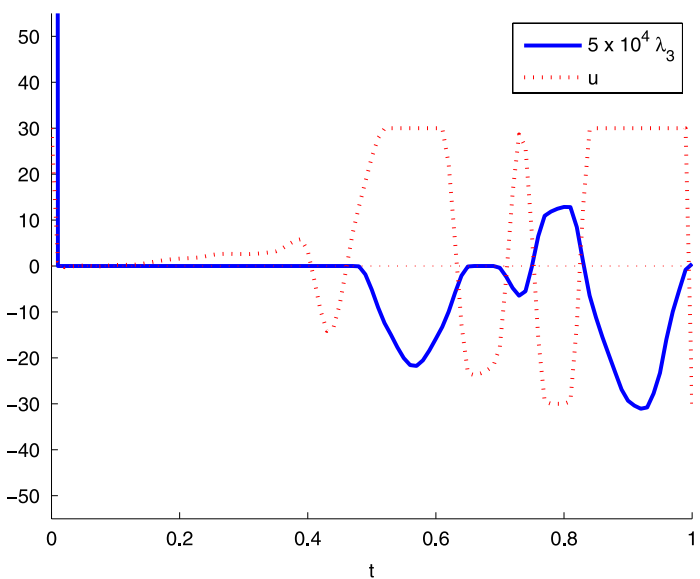

Fig. 2. Switching function $\lambda_{3}$ and optimal control $u$.

probability density of a $\operatorname{Beta}(\alpha, \beta)$ distribution. For a given starting location, the target moves to intercept the HVU with a trajectory determined by the algorithm specified in Ghabcheloo, Kaminer, and Aguiar (2009).

The uncertain optimal control problem is then to determine a control input $u$ which will minimize the probability of not detecting the target subject to the searcher dynamics (23), control constraint, and given initial conditions. As explained in Section 1, the 

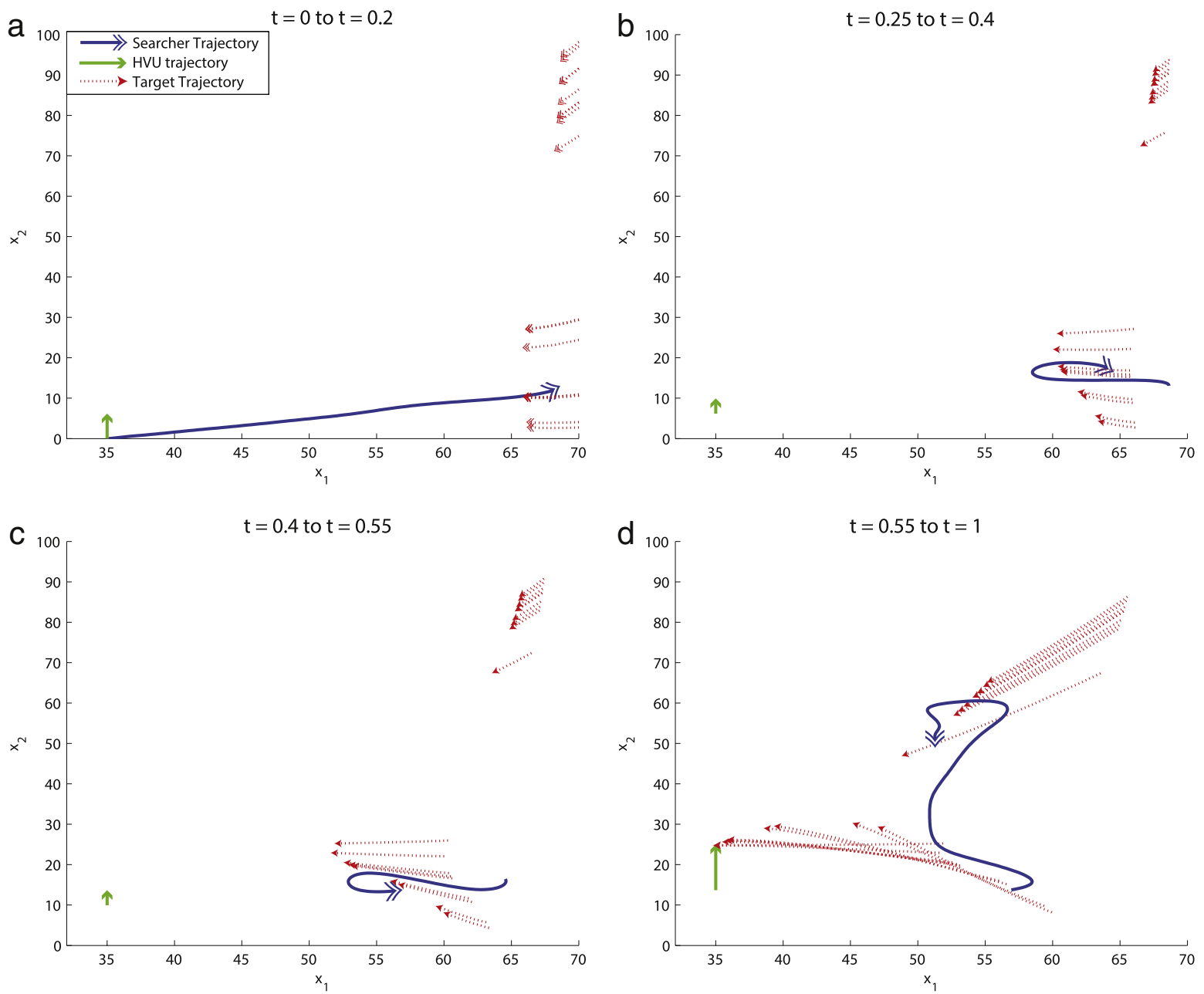

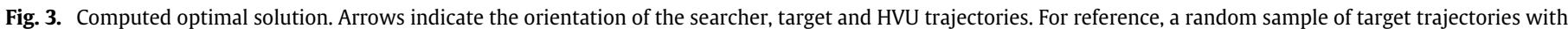
the initial starting location subject to a mixture of beta distributions is shown.

probability of non-detection can be modeled as

$J=\int_{0}^{100} \exp \left(-\int_{0}^{1} \tilde{r}(x(t), y(t, \omega)) d t\right) p(\omega) d \omega$,

where $\tilde{r}$ is the instantaneous rate of detection. The specific form of the detection rate function depends on the sensor. In this example we use the Poisson scan model:

$\tilde{r}(x(t), y(t, \omega))=\beta \Phi\left(\frac{F^{k}-D\|x(t)-y(t, \omega)\|^{2}-b}{\sigma}\right)$,

where $\Phi(\cdot)$ is the standard normal cumulative distribution function, $\|x(t)-y(t, \omega)\|$ is the Euclidean distance between the searcher and the target, $\beta$ is the scan opportunity rate, $F^{k}$ is the so-called "figure of merit" (a sonar characteristic), and $\sigma$ reflects the variability in the "signal excess". In the simulation we use the values $\beta=1.9, F^{k}=120, b=20, D=0.45$, and $\sigma=150$.

The proposed computational framework is applied to this search problem with a LGL quadrature discretization in the parameter space with 42 nodes. Applying this discretization results in a standard optimal control problem which is solved using a pseudospectral discretization scheme in the time domain (Gong, Ross, Kang, \& Fahroo, 2008; Ross \& Karpenko, 2012). The NLP package SNOPT (Gill, Murray, \& Saunders, 2005) is used to calculate the solution to NLP problem produced by this sequence of approximations. This yields a numerical approximation to the optimal trajectory for the searcher.
Remark 5. Note that increasing the number of nodes $M$ improves the accuracy of the approximation of the objective functional; but does not change the dimension of the resulting discretized optimal control problem. However, increasing $M$ requires more target trajectories be stored and increases the computational cost associated to the evaluation of objective functional. Therefore, for problems with a high-dimensional parameter space, the computational cost of the proposed computational framework based on quadrature integration schemes is prohibitive. In Phelps, Royset, and Gong (2013) a discretization scheme for Problem B based on Monte Carlo integration is proposed to avoid the curse of dimensionality for problems with high-dimensional parameter spaces.

Fig. 1 demonstrates the numerical solution obtained by the proposed computational scheme. Snapshots of the searcher and HVU trajectories are shown in Fig. 1(a)-(d). For reference, a random sample of target trajectories with the initial starting location subject to a $\operatorname{Beta}(4,2)$ distribution is also shown. Shown in frame (a), the searcher moves away from the HVU towards the right boundary $x_{1}=70$, as it is known that the target originates at this line. In frames (b)-(d), the searcher, knowing that the target is moving to intercept the HVU, tracks the possible target trajectories back towards the HVU, while adjusting its trajectory so as to match velocity to the target. To assess the validity of the numerical solution, we compute costates, $\lambda_{i}, i=1,2,3$, according to (14)-(15) using the numerical solution $\{x, u\}$. Observe that the control $u$ enters into 
the Hamiltonian only through the linear term $\lambda_{3} u$. Therefore, the Hamiltonian minimization condition (20) implies that

$u(t)= \begin{cases}50, & \text { if } \lambda_{3}(t)<0 \\ -50, & \text { if } \lambda_{3}(t)>0\end{cases}$

In other words, optimal control is of bang-bang type where $\lambda_{3}$ is the switching function. As shown in Fig. 2 the Hamiltonian minimization condition (24) is indeed satisfied.

Next we consider a scenario which differs from the previous scenario only in the initial position of the target. In this scenario, the initial condition of the target is modeled by a mixture of beta distributions, that is, $p(\omega)=p_{12,1}(\omega / 100)+p_{1,12}(\omega / 100)$, where $p_{\alpha, \beta}$ is the probability density of a $\operatorname{Beta}(\alpha, \beta)$ distribution. In this model, at the initial time the target is likely to be near $(70,0)$ or $(70$, $100)$, but significantly less likely to be near $(70,50)$. From the computed optimal searcher trajectory shown in Fig. 3, it is clear that the optimal behavior of the searcher changes depending on the information the searcher has about the starting location of the target. The searcher knows possible target trajectories are very likely to be in one of two groups, one originating near the bottom of the frame and one near the top of the frame. In Fig. 3(a) the searcher, knowing that the target is unlikely to be near the middle of the frame, moves towards the right boundary $x_{1}=70$, but nearer the bottom of the frame. In Fig. 3(b)-(c) the searcher tracks the possible target trajectories back towards the HVU while adjusting its trajectory to match velocity at the target. However, due to the decreasing nature of the detection function, this strategy has diminishing returns. In Fig. 3(d), the searcher leaves the bottom group of possible target trajectories and moves upwards in an attempt to detect the second group of possible target trajectories.

\section{Conclusions}

A computational framework is proposed for a non-standard optimal control problem with an integral over a set of stochastic parameters in the objective functional. The zeroth-order consistency of the numerical scheme is analyzed. In particular, we show that accumulation points of a sequence of solutions of the discretized problem are guaranteed to be optimal solutions of the original uncertain problem. The convergence of the adjoint variables is also demonstrated and a necessary condition that accumulation points must satisfy is derived. The results are demonstrated on a class of optimal search problems with conditionally deterministic target dynamics.

\section{References}

Bedrossian, N., Bhatt, S., Kang, W., \& Ross, I. M. (2009). Zero-propellant maneuver guidance: rotating the international space station with computational dynamic optimization. IEEE Control Systems Magazine, 29(5), 55-73.

Bedrossian, N., Karpenko, M., \& Bhatt, S. (2012). Overclock my satellite. IEEE Spectrum, 49(11), 54-62.

Betts, J. T. (1998). Practical methods for optimal control using nonlinear programming. Journal of Guidance, Control, and Dynamics, 21(2), 193-207.

Bryson, A. E., \& Ho, Y. C. (1975). Applied optimal control. New York: Hemisphere.

Chung, H., Polak, E., Royset, J. O., \& Sastry, S. (2011). On the optimal detection of an underwater intruder in a channel using unmanned underwater vehicles. Naval Research Logistics, 58(8), 804-820.

Cullum, J. (1972). Finite-dimensional approximations of state constrained continuous optimal control problems. SIAM Journal on Control, 10, 649-670.

Ding, X. C., Rahmani, A., \& Egerstedt, M. (2009). Optimal multi-UAV convoy protection. In Proceedings of robot communication and coordination (ROBOCOMM'09). Odense, Denmark

Duchoň, M., \& Maličký, P. (2009). A Helly theorem for functions with values in metric spaces. Tatra Mountains Mathematical Publications, 44, 159-168.

Foraker, J. (2011). Optimal search for moving targets in continuous time and space using consistent approximations (Ph.D. thesis) Monterey, California: Naval Postgradutate School.

Foraker, J., Royset, J. O., \& Kaminer, I. (2014). Optimal search for moving targets in continuous time and space using consistent approximations. Journal of Optimization Theory and Applications, (submitted for publication).
Gadre, A. S., Du, S., \& Stillwell, D. J. (2012). A topological map based approach to long range operation of an unmanned surface vehicle. In American control conference ACC (pp. 5401-5407)

Ghabcheloo, Reza, Kaminer, Isaac, Aguiar, A. Pedro, \& Pascoal, Antonio (2009). A general framework for multiple vehicle time-coordinated path following control. In American controls conference (pp. 3071-3076).

Gill, P., Murray, W., \& Saunders, M. (2005). SNOPT: an SQP algorithm for large-scale constrained optimization. SIAM Review, 47, 99-131.

Gong, Q., Kang, W., \& Ross, I. M. (2006). A pseudospectral method for the optimal control of constrained feedback linearizable systems. IEEE Transactions on Automatic Control, 51(7), 1115-1129.

Gong, Q., Ross, I. M., Kang, W., \& Fahroo, F. (2008). Connections between the covector mapping theorem and convergence of pseudospectral methods for optimal control. Computational Optimization and Applications, 41, 307-335.

Hartl, R., Sethi, S., \& Vickson, R. (1995). A survey of the maximum principles for optimal control problems with state constraints. SIAM Review, 37(2), 181-218.

Hellman, O. (1970). On the effect of a search upon the probability distribution of a target whose motion is a diffusion process. The Annals of Mathematical Statistics, $41,1717-1724$.

Hellman, O. (1972). On the optimal search for a randomly moving target. SIAM Journal on Applied Mathematics, 22, 545-552.

Kameswaran, S., \& Biegler, L. T. (2008). Convergence rates for direct transcription of optimal control problems using collocation at radau points. Computational Optimization and Applications, 41(1), 81-126.

Kang, W. (2010). Rate of convergence for the Legendre pseudospectral optimal control of feedback linearizable systems. Journal of Control Theory and Applications, 8(4), 391-405.

Li, J.-S., Ruths, J., Yu, T.-Y., Arthanari, H., et al. (2011). Optimal pulse design in quantum control: a unified computational method. Proceedings of the National Academy of Sciences, 108(5), 1879-1884

Lukka, M. (1977). On the optimal searching tracks for a moving target. SIAM Journal on Applied Mathematics, 32(1), 126-132.

Mangel, M. (1981). Search for a randomly moving object. SIAM Journal Applied Mathematics, 40(2), 327-338.

Mangel, M. (1982). Probability of success in the search for a moving target Operations Research, 30, 216-222.

Mangel, M. (1989). Search theory: a differential equations approach. In D. V. Chudnovsky, \& G. V. Chudnovsky (Eds.), Search theory: some recent developments (pp. 55-101). New York, NY: Dekker.

Ohsumi, A. (1991). Optimal search for a Markovian target. Naval Research Logistics, 38(4), 531-554

Phelps, C., Gong, Q., Royset, J. O., \& Kaminer, I. (2012). Consistent approximation of an optimal search problem. In Proc. of the 51st IEEE conf. on decision and control (pp. 630-637).

Phelps, C., Royset, J. O., \& Gong, Q. (2013). Sample average approximations in optimal control of uncertain systems. In Proceedings of the 52nd IEEE conference on decision and control (CDC 2013). Florence, Italy.

Polak, E. (1997). Optimization: algorithms and consistent approximations. New York, NY: Springer

Pursiheimo, U. (1976). A control theory approach in the theory of search when the motion of the target is conditionally deterministic with stochastic parameters. Applied Mathematics and Optimization, 2, 259-264.

Quintero, S. A. P., Papi, F., Klein, D. J., \& Chisci, L. (2010). Optimal UAV coordination for target tracking using dynamic programming. In Proceedings of the 49th IEEE conference of decision and control. Atlanta, Georgia.

Ross, I. M., \& Karpenko, M. (2012). A review of pseudospectral optimal control: from theory to flight. Annual Reviews in Control, 36(12), 182-197.

Royset, J. O., \& Sato, H. (2010). Route optimization for multiple searchers. Naval Research Logistics, 57, 701-717.

Ruths, J., \& Li, J. -S. (2010). Optimal ensemble control of open quantum systems with a pseudospectral method. In Proceedings of the 49th IEEE conference on decision and control. Atlanta, Georgia, USA.

Ruths, J., \& Li, J. -S. (2012). Optimal control of inhomogeneous ensembles. IEEE Transactions on Authomatic Control, 57(8), 2021-2032

Saretsalo, L. (1973). On the optimal search for a target whose motion is a Markov process. Journal of Applied Probability, 10, 847-856.

Sato, H., \& Royset, J. O. (2010). Path optimization for the resource-constrained searcher. Naval Research Logistics, 57, 422-440.

Schwartz, A., \& Polak, E. (1996). Consistent approximations for optimal control problems based on Runge-Kutta integration. SIAM Journal on Control and Optimization, 34(4), 1235-1269.

Stone, L. (1977). Search for targets with generalized conditionally deterministic motion. SIAM Journal on Applied Mathematics, 33, 456-468.

Zabarankin, M., Uryasev, S., \& Pardalos, P. (2002). Optimal risk path algorithms. Applied Optimization, 66, 273-296.

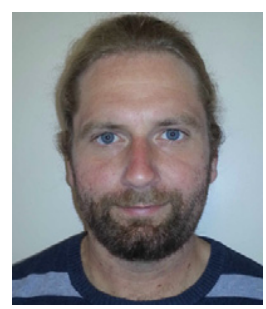

Chris Phelps received a B.S. in Mathematics and Physics in 2007 from the University of California, Santa Barbara, as well as an M.A. in Mathematics in 2010 and Ph.D. in Applied Mathematics and Statistics in 2014 from the University of California, Santa Cruz. His research interests include computational optimal control, optimal control of uncertain systems, and optimal search theory. 


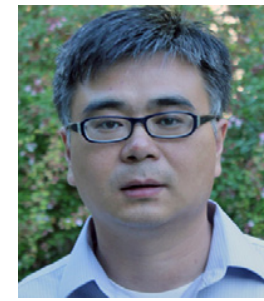

Qi Gong received the B.E. degree in Automation from Dalian University of Science and Technology, China, in 1996, the M.E. degree in Automatic Control from Southeast University, China, in 1999, and the Ph.D. in Electrical Engineering and Computer Science from Case Western Reserve University in 2004. He is currently an Associate Professor in the Department of Applied Mathematics and Statistics at University of California, Santa Cruz. His research interests are in computational optimal control, trajectory optimization, nonlinear control systems, and industry contro applications. He received Postdoctoral Research Associateship Award from National Research Council in 2004.

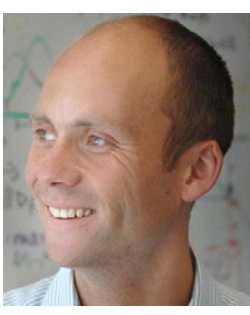

Johannes O. Royset is Associate Chair of Research and Associate Professor of Operations Research at the Naval Postgraduate School. His research focuses on formulating and solving stochastic and deterministic optimization problems arising in data analysis, sensor management, and reliability engineering. He has a Doctor of Philosophy degree from the University of California at Berkeley (2002). He was awarded a National Research Council postdoctoral fellowship in 2003, a Young Investigator Award from the Air Force Office of Scientific Research in 2007, and the Barchi Prize as well as the MOR Journal Award from the Military Operations Research Society in 2009. He received the Carl E. and Jessie W. Menneken Faculty Award for Excellence in Scientific Research in 2010. He is an associate editor of Operations Research, Naval Research Logistics, Journal of Optimization Theory and Applications, and Computational Optimization and Applications.

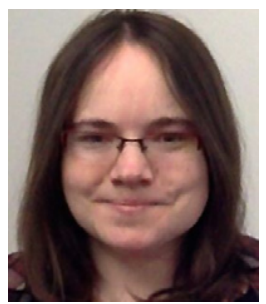

Claire Walton received a B.S. in Mathematics and a B.S. in English from the California Institute of Technology in 2005. She is currently a Ph.D. candidate in Applied Mathematics and Statistics at the University of California, Santa Cruz. Her research interests include computational optimal control, nonlinear trajectory design, and the modeling of multi-agent control problems under uncertainty.

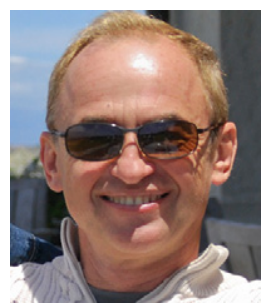

Isaac Kaminer received Ph.D. in Electrical Engineering from University of Michigan in 1992. Before that he spent four years working at Boeing Commercial first as a control engineer in 757/767/747-400 Flight Management Computer Group and then as an engineer in Flight Control Research Group. Since 1992 he has been with the Naval Postgraduate School first at the Aeronautics and Astronautics Department and currently at the Department of Mechanical and Aerospace Engineering where he is a Professor. He has a total of over 20 years of experience in development and flight testing of guidance, navigation and control algorithms for both manned and unmanned aircraft. His more recent efforts were focused on development of coordinated control strategies for multiple UAVs and vision based guidance laws for a single UAV. He has co-authored more than a hundred refereed publications. Over the years his research has been supported by ONR, NASA, US Army, NAVAIR and USSOCOM. 